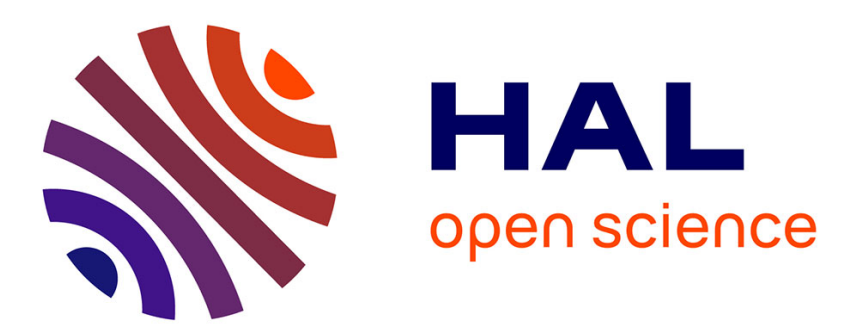

\title{
Neutron diffraction measurements of residual stress distribution in large zirconia based refractory bricks produced by electro-fusion and casting
}

Taylan Örs, Fanny Gouraud, René Guinebretière, Marc Huger, Vincent Michel, Olivier Castelnau

\section{To cite this version:}

Taylan Örs, Fanny Gouraud, René Guinebretière, Marc Huger, Vincent Michel, et al.. Neutron diffraction measurements of residual stress distribution in large zirconia based refractory bricks produced by electro-fusion and casting. Journal of the European Ceramic Society, 2017, 37, pp.2295-2302. 10.1016/j.jeurceramsoc.2017.01.009 . hal-01494338

\section{HAL Id: hal-01494338 \\ https://hal.science/hal-01494338}

Submitted on 23 Mar 2017

HAL is a multi-disciplinary open access archive for the deposit and dissemination of scientific research documents, whether they are published or not. The documents may come from teaching and research institutions in France or abroad, or from public or private research centers.
L'archive ouverte pluridisciplinaire HAL, est destinée au dépôt et à la diffusion de documents scientifiques de niveau recherche, publiés ou non, émanant des établissements d'enseignement et de recherche français ou étrangers, des laboratoires publics ou privés. 


\title{
Neutron diffraction measurements of residual stress distribution in large zirconia based refractory bricks produced by electro-fusion and casting
}

\author{
Taylan Örs ${ }^{a, b}, *$, Fanny Gouraud ${ }^{c}$, René Guinebretière ${ }^{\mathrm{c}, *}$, Marc Huger ${ }^{\mathrm{c}}$, Vincent Michel ${ }^{\mathrm{a}}$, \\ Olivier Castelnau ${ }^{\mathrm{a}}$ \\ a PIMM, UMR CNRS 8006, Arts and Métiers ParisTech, CNAM, 151 Bd de l'Hopital, 75013 Paris, France \\ b LEM3, UMR CNRS 7239, Université de Lorraine, Ile du Saulcy, 57045 Metz Cedex 1, France \\ ' ENSCI, SPCTS, UMR CNRS 7315, 12 rue Atlantis, 87068 Limoges, France
}

\section{A R T I C L E I N F O}

\section{Article history:}

Received 26 September 2016

Received in revised form 4 January 2017

Accepted 6 January 2017

Available online 17 January 2017

\section{Keywords:}

Residual stresses

Zirconia

Neutron diffraction

Refractory materials

\begin{abstract}
A B S T R A C T
Electro-fusion and casting is used to produce large refractory bricks ( $\sim 250 \mathrm{~kg})$ containing a high amount of $\mathrm{ZrO}_{2}$. These bricks are used in glass-making furnaces where good mechanical performance is required at very high temperatures $\left(>1500^{\circ} \mathrm{C}\right)$. During the manufacturing procedure, they develop large residual stresses as a result of the cooling conditions and structural phase transformations they underwent. This leads to stress concentration and crack formation at different length scales. In order to characterize these phenomena, a 'multi-scale' analysis approach is under development, where different internal strain measurement methods are combined. In this approach we benefit from different gauge volumes provided by various diffraction methods, ranging from a few hundred nanometres to a few tens of millimetres. In the present paper, the results of neutron diffraction measurements on large $\mathrm{ZrO}_{2}$ blocks are given. These results show the level of internal strains at the millimetre scale, based on ( $\overline{3} 11)$ reflection of the monoclinic $\mathrm{ZrO}_{2}$. Overall, a range of $0.025 \%$ tensile to $0.1 \%$ compressive strain was observed. Clear strain gradients were also visible, as larger values in the interior of the block were encountered.
\end{abstract}

\section{Introduction}

Refractory materials containing high amounts of $\mathrm{ZrO}_{2}$ are widely used as flux blocks for glass-making furnaces owing to the resistance of $\mathrm{ZrO}_{2}$ to the corrosive effects of molten glass [1,2]. The size of these blocks is several hundreds of millimetres. In order to fully benefit from the corrosion resistance, a lower degree of open porosity is required. Therefore, the large $\mathrm{ZrO}_{2}$ blocks are produced by electro-fusion followed by casting. However, during the manufacturing process, thermal gradients, successive structural phase transitions (SPTs), and anisotropic thermal contraction of zirconia induce the development of significant amounts of internal residual stresses [3,4].

Under ambient pressure, pure zirconia undergoes three SPT during cooling from its melt [5]. It solidifies into a cubic crystal structure $\left(\mathrm{c}-\mathrm{ZrO}_{2}\right.$, space group $\left.\mathrm{Fm} \overline{3} \mathrm{~m}\right)$ at about $2700^{\circ} \mathrm{C}$; upon

\footnotetext{
* Corresponding authors.

E-mail addresses: taylanors@gmail.com (T. Örs), rene.guinebretiere@unilim.fr (R. Guinebretière).
}

cooling to $2300{ }^{\circ} \mathrm{C}$ it transforms to tetragonal ( $\mathrm{t}-\mathrm{ZrO}_{2}$, space group $\mathrm{P}_{2} / \mathrm{nmc}$ ), and finally it becomes monoclinic ( $\mathrm{m}-\mathrm{ZrO}_{2}$, space group $P 2_{1} / c$ ) around $1170^{\circ} \mathrm{C}$. This last SPT is a first order transition and is of martensitic type. Moreover, it induces a large volumetric expansion which, in the absence of any stresses, is typically close to 4.5\%. Therefore the tetragonal-monoclinic SPT alone creates large amounts of strain in the material and induces cracking, which finally leads to breaking the pure bulk zirconia to pieces. In the case of refractory blocks containing a large volume fraction of zirconia, an amorphous matrix phase is used between the $\mathrm{ZrO}_{2}$ crystals and acts as a medium for partial stress relief [1].

Because of the stress effect, the actual temperatures of the SPTs between tetragonal to monoclinic phases (both forward and backward transformations) are usually very different from that predicted for a perfect free single crystal. The transition of a single crystal from one state to the other occurs when the sign of the free energy balance between the two phases changes. This depends mainly on the temperature, the size of the considered crystal, and the energy associated with the interfacial strain. In a zirconia block containing a very large number of zirconia crystals (having various sizes and crystal orientations), the tetragonal-monoclinic SPTs 
occur thus in a large temperature range, and a hysteresis effect is observed both during the heating and cooling processes of the sample. Similar to what is observed in steels, this martensitic transition generally occurs at a lower temperature during the cooling than during the heating, and in some cases, residual tetragonal zirconia can be observed at room temperature.

Substantial efforts on the numerical modelling of these stresses have been ongoing for two decades $[3,4,6]$. Nevertheless, since the causes for the internal stresses are numerous, with each of them involving different but co-dependent phenomena, these models do not yet account for all deformation mechanisms and therefore are not yet predictive. In order to rigorously analyse the stress build-up within these $\mathrm{ZrO}_{2}$ blocks, an experimental multi-scale approach is being developed in which we combine neutrons, X-rays and electron diffraction. The global scale, including the contribution of a large number of crystals, is explored by both room temperature neutron diffraction and in-situ high-temperature X-ray diffraction experiments. The strain state is also probed at the very local scale through Laue microdiffraction and electron back scatter diffraction (EBSD). Thus the used diffraction volumes range from a few hundreds of nanometres to a few tens of millimetres. These different diffraction gauge volume sizes enable averaging over different scales and thus facilitate the analysis of the effects of different causes of stress on the system.

In this paper, we discuss the results of the first part of this analysis: the residual strains at millimetre scale as measured by neutron diffraction. Two different blocks of high zirconia content were prepared by Saint-Gobain CREE. These two blocks differ mainly in the composition of the amorphous matrix phase around the zirconia crystals.

Neutron diffraction is a widely used technique for the measurement of the residual stress of crystalline matter on larger scales. Even though hard X-rays from high brilliance synchrotron sources are emerging as strong alternatives [7], neutrons still perform better at high penetration. A good review for the comparison of the two methods is given in [8]. Since neutron beams are less affected by intensity loss at greater depths than are X-ray beams, neutron diffraction is generally the method of choice when it comes to large engineering pieces. There is a vast literature on strain measurements made by neutron diffraction on large industrial metallic pieces [9-12]. However, to the best of our knowledge, there seems to be a clear lack of residual stress-strain studies with diffraction methods on ceramic materials produced at this scale. The only neutron measurements on refractory blocks with such a high zirconia content was done for the purpose of texture analysis [13]. Therefore the significance of the present paper is also that it is among the first in the field of residual strain analysis of an industrial size ceramic refractory material.

\section{Experimental}

\subsection{Sample preparation and microstructure}

Two blocks of different compositions were prepared and cast by the company Saint-Gobain CREE. These compositions are exactly the same as those reported in [13]. They therefore both contain $94 \mathrm{wt} . \% \mathrm{ZrO}_{2}$. The remaining content (6 wt.\%) is alkalisilicate type glass for one block (labelled ZS hereafter) and borosilicate glass for the other (ZB hereafter).

Fig. 1a shows the typical microstructure of these materials exemplified by a sample of ZB composition which was cut and polished for SEM imaging after casting. The micrograph reveals large zirconia areas (in grey) formed during the solidification process [14], having sizes of a few hundred micrometres. Each of these areas was initially (i.e. at high temperature) a single crystal with a cubic

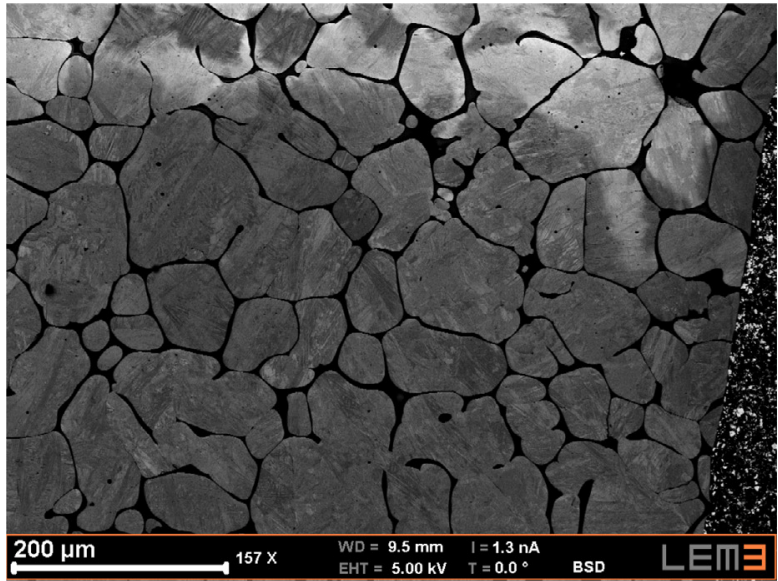

(a)

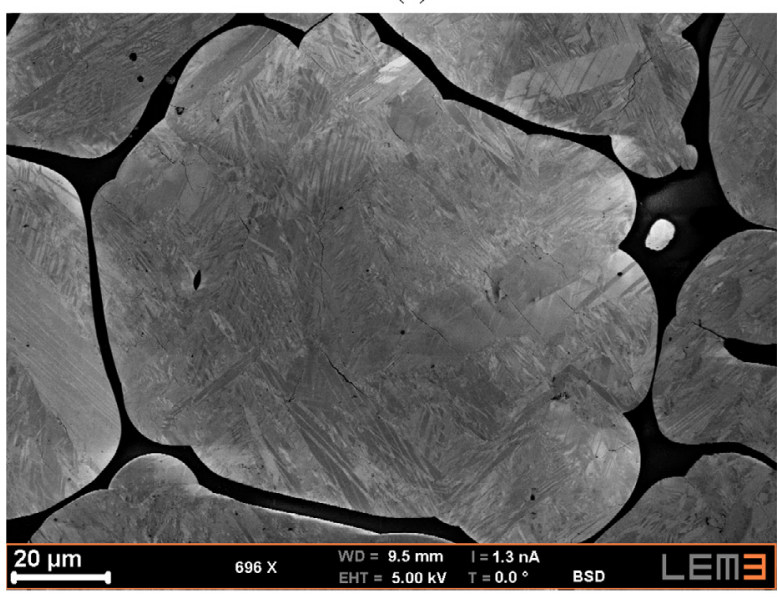

(b)

Fig. 1. Microstructure of the $\mathrm{ZB}$ sample. $\mathrm{ZrO}_{2}$ 'domains' (in grey shades) in a glassy matrix (in black) (a), and a close-up of a single domain, showing the needle- and plate-like monoclinic $\mathrm{ZrO}_{2}$ crystallites (b). The image is taken from a fused-cast sample with the same composition as the measured block sample.

structure [15]. These areas are surrounded by the amorphous silicate phases (in black). As seen in Fig. 1b, they contain a large number of monoclinic $\mathrm{ZrO}_{2}$ crystals which are arranged in specific orientations with respect to each other. The sizes of these crystals range from $50 \mu \mathrm{m}$ down to a few hundreds of nanometres. The EBSD results on these materials [14] confirm a very well defined local texture within the mentioned areas of a few hundred micrometres. This is a result of orientation relationships established by the successive SPTs [15]. The texture at the macroscopic scale is, on the other hand, found to be random [13].

\subsection{Neutron diffraction}

Using neutron diffraction is the best option when one wants to scan very thick specimens up to a few centimetres depth. The neutron diffraction measurements were done at the SALSA beamline of Institute Laue-Langevin (ILL) in Grenoble, France. the $\mathrm{ZrO}_{2}$ blocks were measured in their entirety, i.e. without slicing or sampling. The only treatments made to the blocks were removing the riser (masselotte) and machining the surface to decrease the surface roughness.

The final dimensions were $437 \times 240 \times 305 \mathrm{~mm}^{3}$ for the block of composition ZB and $500 \times 102 \times 688 \mathrm{~mm}^{3}$ for the block of composition ZS along the $x$-, $y$ - and $z$-directions of the reference system in Fig. 2. A schematic view of the ZB block is also given in 


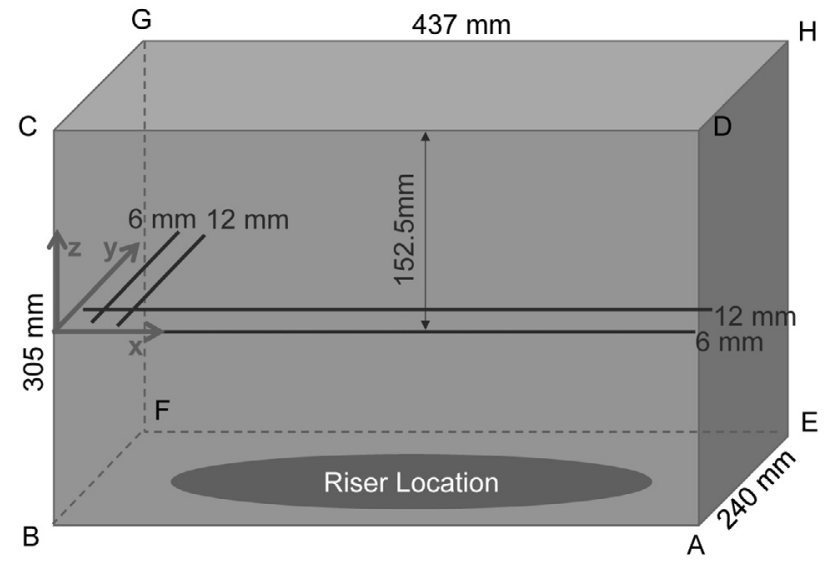

Fig. 2. Schematic representation of the ZB block. The block was positioned upside down with respect to the casting direction, i.e. the riser was at the bottom before its removal. In the middle of the $[C B]$ segment, a reference system is introduced which will be used throughout the paper. This reference system is attached to the block, assigning the following directions: casting direction ( $\mathrm{C}$ to $\mathrm{B}$ ) is $-\vec{z}$, towards interior of the block along the longer edge is $+\vec{x}$, and towards interior of the block along the shorter edge is $+\vec{y}$. The origin is the midpoint of $[\mathrm{CB}]$. The same convention is used for the ZS sample. The black lines indicate the measured horizontal lines measured parallel to the large (ABCD) and small (BFGC) faces. They correspond to the scans named ' $Z \mathrm{~B} x$-line 6', 'ZB $x$-line 12', 'ZB $y$-line 6' and ' $Z \mathrm{~B} y$-line 12' in Table 1 . The scans labelled 'Corner $x$-dir' and 'Corner $y$-dir' in the same table are measured around the corner where the reference system is centred.

Fig. 2 in its measurement configuration. Each one of the measured blocks, weighing approximately $250 \mathrm{~kg}$, was mounted on a hexapod which allows rotating and translating the block along all the spatial directions. The ZB block fixed on the hexapod is shown in Fig. 3.

The measurements were done at a fixed position of the $2 \mathrm{D}$ detector leading to a range of diffraction angles $2 \theta$ between $55^{\circ}$ and $70^{\circ}$. The gauge volume is defined by the intersection of two collimators. The primary collimator defines the incoming beam arriving from the monochromator to the sample and determines where the diffraction will occur within the sample. The secondary (radial) collimator leads the diffracted beam to the pixel detector, thereby determining the exact limits and the $2 \theta$ range of the part of the diffracted beam to be considered. This is illustrated in the inset of Fig. 3. Accordingly, the area of the horizontal crosssection of the diffraction volume was $4 \times 4 \times \sin 2 \theta \mathrm{mm}^{2}$. The height of the gauge was $20 \mathrm{~mm}$. The wavelength was set to 1.644 Åfor the entire experiment (the attenuation length of $\mathrm{ZrO}_{2}$ for neutrons at 1.644 Åis $2000 \mathrm{~mm}$ [16]). The counting of the neutrons per given detector position was done either until 50,000 total neutron counts were received by the detector or for $1 \mathrm{~h}$, whichever condition was reached earlier.

The choice of the $(h k l)$ Bragg peak to be measured is not trivial. First of all, due to its monoclinic structure, the powder pattern of $\mathrm{ZrO}_{2}$ contains a large number of diffraction peaks with possible overlaps (Fig. 4). Next, based on Eq. (2), the resolution in strain increases quickly with the Bragg angle, and therefore large $2 \theta$ values must be favoured. Next, the gauge volume is smaller and its position better defined for $2 \theta$ close to $90^{\circ}$. And lastly, the modest resolution of neutron diffractometers amplifies the overlap of the peaks, which can be an issue for data processing. Based on these constraints, the experiment was done using the peak around $2 \theta=61^{\circ}$, which contains information on the (311) reflection with weak contributions from overlapping (310) and (212) reflections (Fig. 4, see insets (a) and (b)). The theoretical intensity of the (311) reflection is approximately 25 times greater than that of (310) and 600 times greater than that of (212). Therefore, the contribution of these two small peaks, (310) and (212), will be

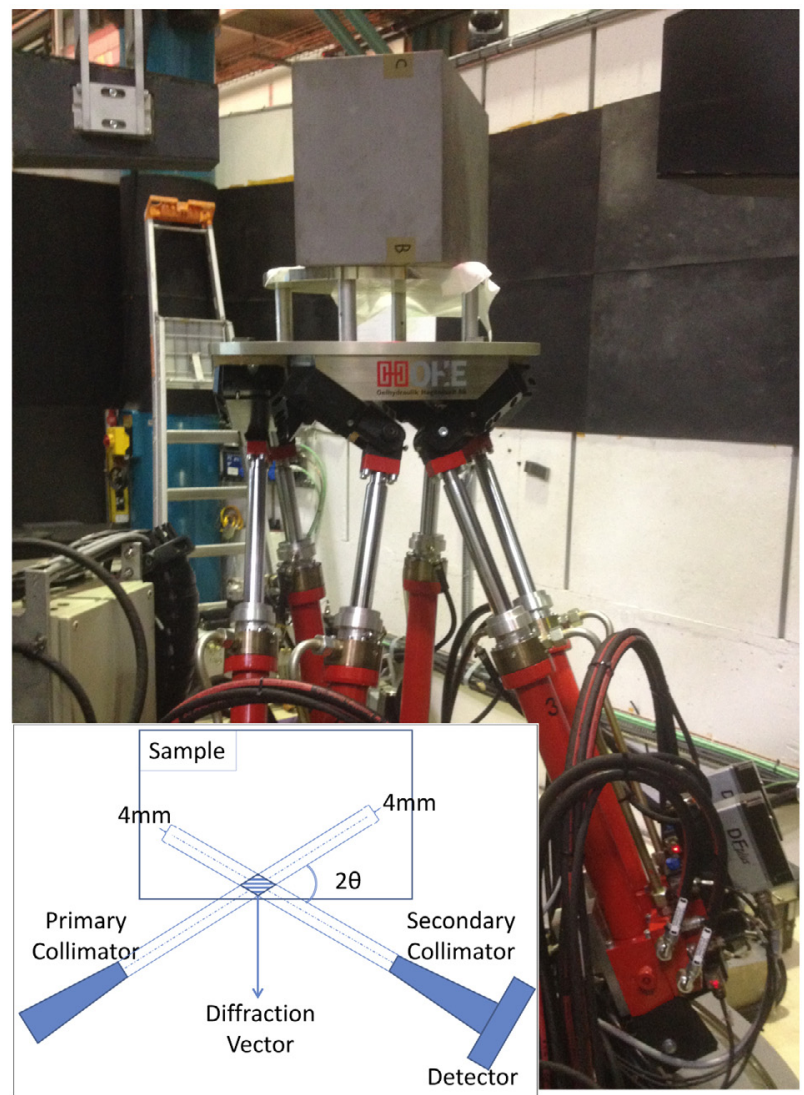

Fig. 3. Main parts of the SALSA instrument. The neutron beam arrives on the sample through the primary collimator (on the left). The sample is located on top of a hexapod and can be rotated and translated around multiple axes. The diffraction volume is defined by the second collimator (on the right-hand side of the image) which directs the incoming beam to the detector (not shown). Inset: Projection of the diffraction volume (shaded area) on the horizontal plane, defined by the two collimators.

neglected, and the peak measured at $2 \theta \simeq 61^{\circ}$ is attributed solely to ( $\overline{3} 11)$.

As for the initial treatment of the measured diffraction images (image reading out and format conversion), LAMP software written by the ILL staff was used [18]. For radial integration of the 2D diffraction patterns, a software called DataGUInz [19] was used. After integration, a correction was applied to the data to remove the possible effects of the radial collimator foils on the diffracted beam intensity. Flat field images obtained from an amorphous material, e.g. plexiglass, were used for this purpose.

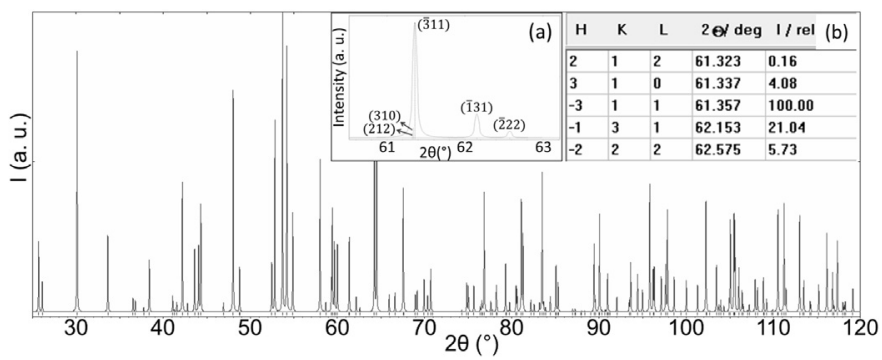

Fig. 4. Complete simulated powder diffraction pattern of $\mathrm{m}-\mathrm{Z} \mathrm{rO}_{2}$. Wavelength used for the simulation is equal to the one used for the experiments (1.644 $\AA$ ). The calculation was performed by PowderCell software [17]. Inset (a): The reflection used during the strain measurement, $(\overline{3} 11)$, in detail with the overlapping and nearby reflections. Inset (b): Positions and relative intensities of the reflections in the range shown in inset (a). 


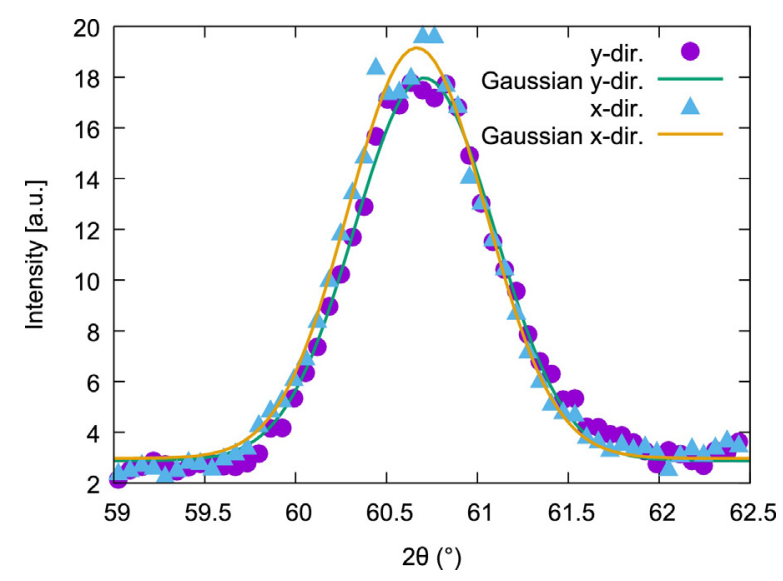

Fig. 5. Comparison of the Gaussian fits of the ( $\overline{3} 11)$ peaks acquired at a given position in the ZB block for two different directions of the diffraction vector (thus strain). These directions are indicated with respect to the reference system introduced in Fig. 2.

\subsection{Peak fitting and strain calculation}

In order to obtain the exact $2 \theta$ positions of the diffraction peaks, the data were fitted by a Gaussian function

$I(2 \theta)=\frac{a}{s \sqrt{2 \pi}} e^{-\frac{(2 \theta-\overline{2} \theta)^{2}}{2 s^{2}}}+B$

where $2 \theta$ is twice the diffraction angle, $I(2 \theta)$ is the intensity value (in arbitrary units) at a given $2 \theta, 2 \theta$ is the mean value of $2 \theta$ in degrees, $s$ is the standard deviation of $2 \theta, B$ is the constant background noise intensity and $\frac{a}{s \sqrt{2 \pi}}+B$ is the intensity (in arbitrary units) of the maximum. Employing this formula, a single maximum for each peak area was calculated. The fits were calculated by the gnuplot [20] software. Examples of fits are shown in Fig. 5.

Scanning deeper into our materials sometimes leads to a severe loss of diffracted beam intensity and noisy data. As a result, it becomes difficult to determine the exact positions of the maxima. Therefore we used criteria for reliable peak identification. For this purpose, a signal-to-noise ratio was defined as a/B for the parameters of the Gaussian fit function in Eq. (1). In this study, a minimum signal-to-noise ratio of 2.5 and a maximum value for fitting errors on $2 \theta$ (denoted by $u_{2 \theta}^{-}$) of $0.015^{\circ}$ were defined for a peak to be declared reliable.

The reference peak positions were determined by measuring small cubes of $10 \times 10 \times 10 \mathrm{~mm}^{3}$ cut from corners of the blocks during the removal of the riser. The cutting was done for the purpose of producing a sample which is free of stress at the scale of interest for this measurement, i.e. at the size of the gauge volume, as there was no macroscopic stress applied to those cubes. Two cubes for each block composition were obtained. Two measurements were taken from each cubic reference, and the measured peak positions were averaged for each block. These reference samples yielded $\overline{2} \theta_{0}=60.682(8)^{\circ}$ for the $(\overline{3} 11)$ peak of $\mathrm{ZB}$ composition, and $2^{-} \theta_{0}=60.705(25)^{\circ}$ for $\mathrm{ZS}$.

The strain calculation was done according to the following standard formula, which is obtained by incorporating Bragg's law into the definition of strain:

$\varepsilon=\frac{\left(d-d_{0}\right)}{d_{0}}=-\frac{1}{2} \cot \bar{\theta}_{0}(\Delta 2 \theta)$

where $\Delta(2 \theta)$ is the difference between a given reflection measured at a given $(x, y, z)$ coordinate and the reference value measured from the cubes and for this reflection, i.e. $2 \theta-2 \theta_{0}, d$ is the measured mean interplanar spacing for the given reflection at a measurement point $(x, y, z)$ on the block and $d_{0}$ is the value of the same mean interplanar spacing in the stress-free condition obtained from cut reference samples. The standard measurement errors were calculated by propagation of errors as discussed in Appendix A.

\section{Results}

\subsection{ZB block}

Examples of Gaussian fits of the diffraction patterns are given in Fig. 5 for the $(\overline{3} 11)$ reflections of two different measurements of the ZB block. A corner of this block close to the middle of the [CB] edge (as named in Fig. 2) with the coordinates of $x=10 \mathrm{~mm}$, $y=6 \mathrm{~mm}, z=0 \mathrm{~mm}$ has been measured with two different directions of the diffraction vector $(\vec{x}$ and $\vec{y})$. This was obtained by rotating the block $90^{\circ}$ between two measurements around the centre of the diffraction volume about an axis parallel to $\vec{z}$ ( $\omega$-axis). Therefore the resulting diffraction volumes in these two conditions are very similar. A direct comparison of the two yields the difference in strain values along two different principal directions at a given point. The measured strain at this point is tensile along the $x$ direction $(0.023 \% \pm 0.015 \%)$ and compressive along the $y$-direction $(-0.039 \% \pm 0.015 \%)$. Using the same idea, strain measurements along the $x$ - and the $y$-directions were realized for a mesh with $x=10-40 \mathrm{~mm}$ and $y=6-42 \mathrm{~mm}$ with step sizes of $10 \mathrm{~mm}$ along $\vec{x}$ and $6 \mathrm{~mm}$ along $\vec{y}$. The third dimension was kept constant at $z=0$. These scans are labelled 'Corner $x$-dir' and 'Corner $y$-dir' in Table 1 according to their strain measurement direction, i.e. the direction of the diffraction vector. The 2-dimensional strain maps generated as a result are given in Fig. 6.

The two horizontal lines parallel to the large face of ZB as shown in Fig. 2 and detailed in Table 1 under the names ' $Z \mathrm{~B} x$-line 6 ' and 'ZB $x$-line 12' were measured as scans along the $x$-direction. The results are shown in Fig. 7a. For these scans, the diffraction vector lies perpendicular to the large surface of the block. We have also tried doing the same scan but for a diffraction vector parallel to the surface, i.e. in transmission geometry, but the attenuation of the beam by the whole block thickness was too strong to obtain acceptable signal-to-noise ratios.

Another set of two horizontal lines, this time parallel to the smaller face, were measured with a $y$-range of $10-230 \mathrm{~mm}$ (' $\mathrm{ZB}$

Table 1

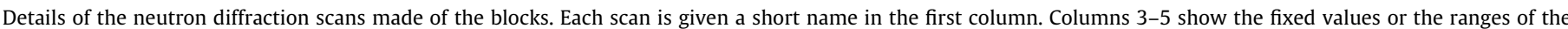

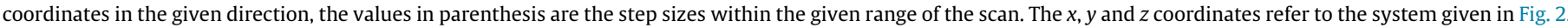

\begin{tabular}{|c|c|c|c|c|c|c|c|}
\hline Scan name & Block & $x$ in $\mathrm{mm}(\mathrm{mm} / \mathrm{step})$ & $y$ in $\mathrm{mm}(\mathrm{mm} / \mathrm{step})$ & $z$ in $\mathrm{mm}(\mathrm{mm} / \mathrm{step})$ & Reflection & Diffraction vector & In Fig. \\
\hline Corner $x$-dir & ZB & $10-40,(10)$ & $6-42,(6)$ & 0 & $(\overline{3} 11)$ & $\vec{x}$ & $6 a$ \\
\hline Corner $y$-dir & ZB & $10-40,(10)$ & $6-42,(6)$ & 0 & $(\overline{3} 11)$ & $\vec{y}$ & $6 b$ \\
\hline ZB $x$-line 6 & ZB & $10-430,(10)$ & 6 & 0 & $(\overline{3} 11)$ & $\vec{y}$ & $7 a$ \\
\hline ZB $x$-line 12 & ZB & $10-430,(10)$ & 12 & 0 & $(\overline{3} 11)$ & $\vec{y}$ & $7 a$ \\
\hline ZB y-line 6 & ZB & 6 & $10-230,(10)$ & 0 & $(\overline{3} 11)$ & $\vec{x}$ & $7 \mathrm{~b}$ \\
\hline ZB $y$-line 12 & ZB & 12 & $10-230,(10)$ & 0 & $(\overline{3} 11)$ & $\vec{x}$ & $7 b$ \\
\hline ZS $x$-line 6 & ZS & $12.5-487.5,(25)$ & 6 & 0 & $(\overline{3} 11)$ & $\vec{y}$ & 8 \\
\hline ZS $x$-line 12 & ZS & $12.5-487.5,(25)$ & 12 & 0 & $(\overline{3} 11)$ & $\vec{y}$ & 8 \\
\hline
\end{tabular}




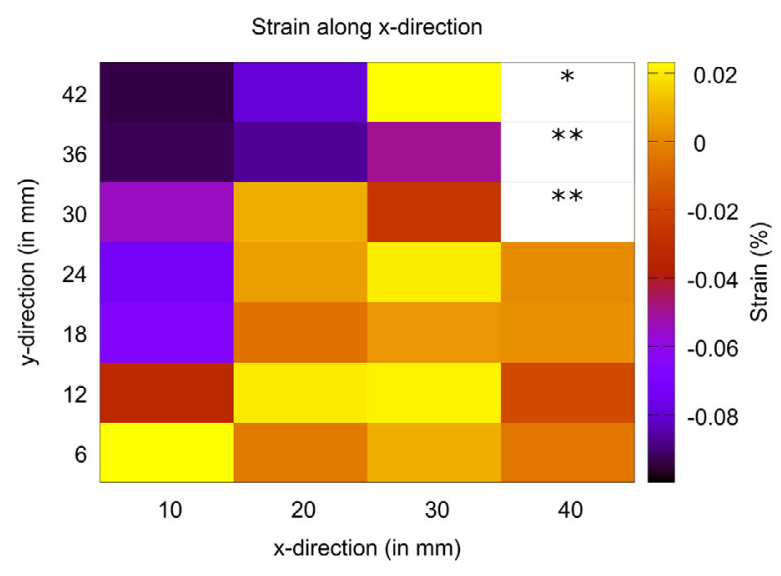

(a)

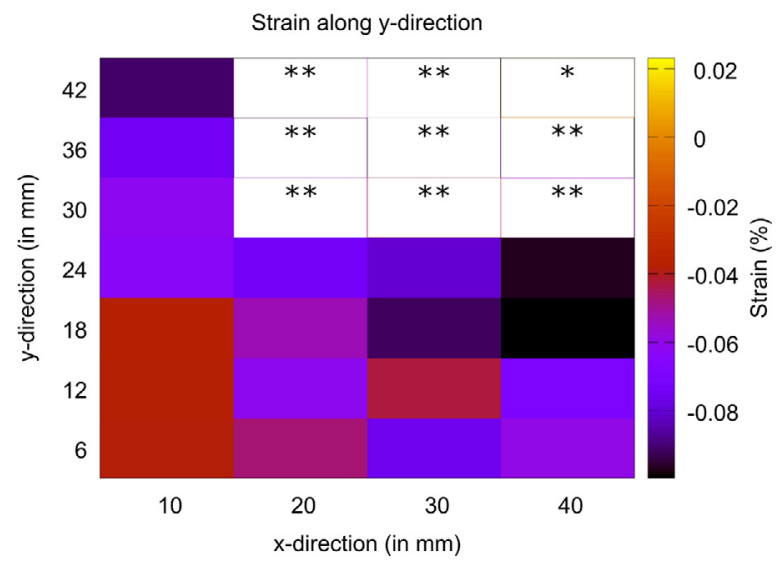

(b)

Fig. 6. Strain maps for the measured mesh of $x=10-40 \mathrm{~mm}, y=6-42 \mathrm{~mm}$ and $z=0 \mathrm{~mm}$ for the strain (a) along the $x$-direction ('Corner $x$-dir' in Table 1) and (b) along the $y$-direction, 'Corner $y$-dir'. The measurement was based on the position of the (311) reflection. *: Skipped point due to excessive acquisition time, ${ }^{* *}$ : Measured but rejected due to bad signal-to-noise ratio and/or large fitting error $u_{\overline{2} \bar{\theta}}$.

$y$-line 6' and ' $Z \mathrm{~B}$ y-line 12' in Table 1 ). Fig. 7b shows the resulting strain graphs with varying $y$-positions.

\subsection{ZS block}

The ZS block $\left(688 \times 500 \times 102 \mathrm{~mm}^{3}\right)$ was placed in an equivalent way to the $\mathrm{ZB}$ one, i.e. on top of the cut surface where the riser was once located. In this configuration, lines parallel to the large face were measured as shown in Table 1 as ' $\mathrm{ZS} x$-line 6' and 'ZS $x$-line 12 '. The reflection was chosen to be $(\overline{3} 11)$ as in the previous cases. The strain evolutions along these horizontal lines are given in Fig. 8.

We note that for both ZB and ZS blocks it would have been interesting to observe the evolution of strain in the $z$-direction. However, since the acquisition of reliable neutron diffraction data for strain measurement purposes requires long exposure time, this measurement could not be realized during our allocated experimental time. Moreover, measuring the stress in 3D would have required either cutting the specimen or rotating it. Cutting the specimen relaxes the internal stresses that we aimed to measure, so this possibility was not considered. On the other hand, for such heavy samples $(250 \mathrm{~kg})$ the use of a hexapod was necessary and therefore sample rotation was limited.

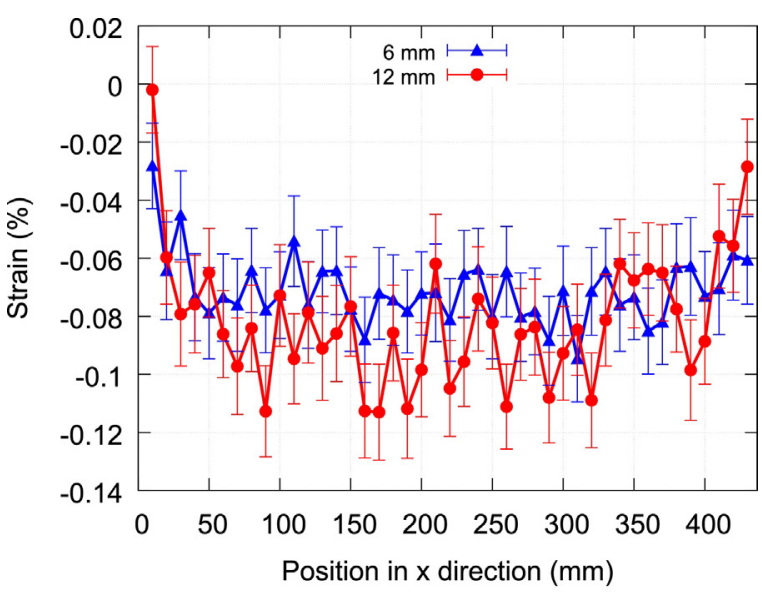

(a)

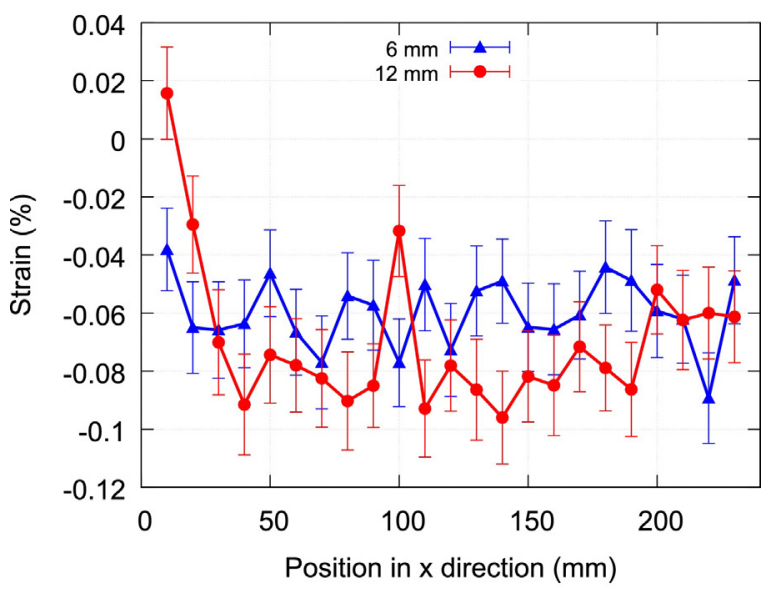

(b)

Fig. 7. Horizontal lines measured in the ZB sample. (a) Results of the ' $Z B$ x-line 6' (blue) and ' $Z B x$-line 12' (red) scans in Table 1; (b) 'ZB $y$-line 6' (blue) and ' $Z B$-line 12 ' (red) scans in Table 1. Error bars are calculated with the method of propagation of errors discussed in Appendix A. (For interpretation of the references to colour in this figure legend, the reader is referred to the web version of the article.)

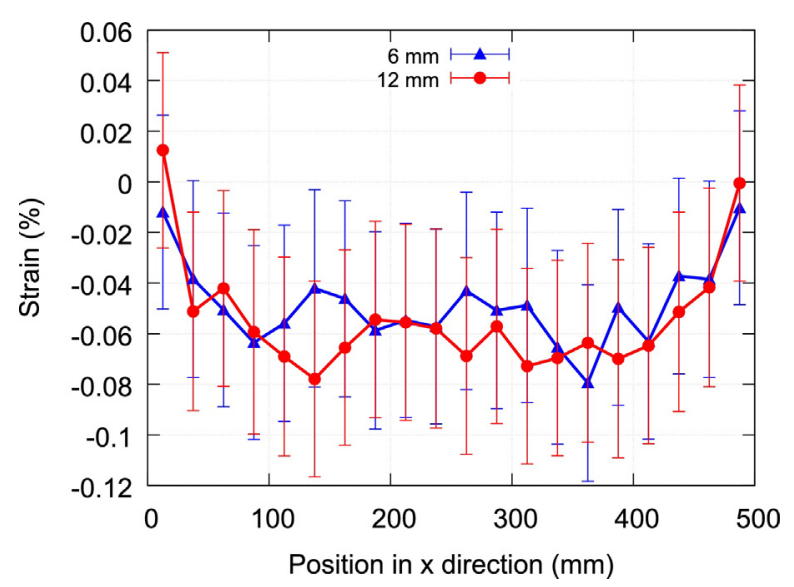

Fig. 8. Results of the 'ZS $x$-line 6' (blue) and 'ZS $x$-line 12' (red) scans specified in Table 1. (For interpretation of the references to colour in this figure legend, the reader is referred to the web version of the article.) 


\section{Discussion}

According to the results of the 'Corner $x$-dir' and 'Corner $y$-dir' scans in Fig. 6, the strain along the $y$-direction in this region is more compressive than the strain along the $x$-direction. There seems to be a slight tendency of increasing strain with depth for both of the directions.

For both of the ' $Z B \mathrm{~B} x$-line 6' and ' $\mathrm{ZB} x$-line 12' scans (Fig. 7a), clear gradients are observed from the relaxed near-zero level deformation at the surface to up to $0.1 \%$ compressive strain in the interior parts. The state of deformation looks symmetric with respect to the centre of the line. One can also see that at a depth of $12 \mathrm{~mm}$, the compressive stress becomes more pronounced.

For the lines labelled ' $\mathrm{ZB} y$-line 6' and ' $\mathrm{ZB} y$-line 12', a similar gradient is observed in one side $(y=0)$ of the block although this is not clear for the other end $(y=240)$. Just as for the earlier set of two horizontal lines, there is a clear tendency in higher compressive strain at a depth of $12 \mathrm{~mm}$.

In order to understand the strain results presented, one needs to look more into the details of the effects of the processing of these materials. As discussed earlier, there are various reasons for the build-up of internal stresses. One reason is the thermal gradients that occur during casting. Stresses due to such gradients are commonly encountered in the casting of metallic materials as well [21]. However, in the case of ceramics the gradients are expected to be much higher, mainly due to the lower thermal conductivity of such materials. The main principle for this type of stress build-up is that the different temperatures (and cooling rates) in the interior and the exterior lead to different thermal expansions at different times during the casting.

In zirconia based materials, thermal gradients during casting could play yet another role directly linked to the tetragonal to monoclinic phase transition. The stress field established before the martensitic phase transformations determines the transition temperature of a given region within the block. The starting point of the martensitic tetragonal to monoclinic SPT is then dependent on some processing parameters but it is generally below $1000^{\circ} \mathrm{C}$ [14]. At such a temperature, the discrepancy between the internal and external temperature of the block is typically lower than $100^{\circ} \mathrm{C}$ (see fig. 11 in [6]). Such a temperature gap is indeed small but since the phase transformation of a given individual crystal is also related to its size and to the stresses applied on it, the thermal gradients might indirectly affect its final stress state. This complicated mechanism causes a final stress field that has to be studied in more detail.

The cooling conditions during casting are assumed to be symmetric with respect to the centre along the $x$ - and $y$-directions. This assumption is used within thermo-mechanical modelling studies on blocks which simulate the conditions during their production [6]. The symmetry of the strain gradient seen in Fig. 7a supports this assumption.

As mentioned earlier, the scale of the predicted residual strain varies from the sub-micron to the millimetre level. One would expect to see smaller values of strain from averaging over larger volumes. However, the current residual strain analysis at the scale of a few millimetres yields already a significant amount of deformation. This could be a hint that at the sub-micron scale, the strain values are quite high.

The high deformation values at this millimetre scale persist even in regions right below the surface (6-12 mm). However, for the reasons given in Section 2.3 (low signal-to-noise ratio and larger fitting errors), it was not possible to measure the deeper parts of the blocks to observe the evolution of the strain.

For the ZS block, the 'ZS $x$-line 6' and 'ZS $x$-line 12' scan results in Fig. 8 indicate the following: The more-or-less symmetrical gradient from the surface to the inner parts of the block is again clearly visible and the deformation values are generally within the range of $(-0.1 \%,+0.02 \%)$, similar to ZB. The difference between the two different depths ( $y$-values) are however, less pronounced.

According to these results, despite the different compositions and different aspect ratios of the two blocks (ZB and ZS), their internal stress build-up at this scale seems similar. It is worth mentioning that the larger error bars in ZS measurements in general are due to larger differences in the measurement of the two reference samples for this composition $\left(u_{2 \bar{\theta}_{0}}=0.025^{\circ}\right)$, as reported earlier in Section 2.

So far, only the values of the elastic strain have been reported, as these are the direct outputs of diffraction measurements. These elastic strain values will allow a quantitative comparison with thermo-mechanical models, in the future. It can however be of interest to estimate the level of stress each gauge volume is subjected to. In order to do that, a scale transition approach is required, for two reasons [22-25]. First, the intersection between the incident and diffracted beams defines the gauge volume (denoted $V$ ), as depicted in Fig. 3. But within this gauge volume, only grains that satisfy the Bragg conditions, i.e. with the ( $\overline{3} 11)$ plane perpendicular to the diffraction vector, really contribute to the diffracted peak. The total volume of those grains (called the 'diffraction volume', $\Omega$ ) is significantly smaller than $V$, and does not provide a representative volume element (RVE) for $V$. Second, the measured lattice strain defined in Eq. (2) is a projection of the mean elastic strain tensor within the diffraction volume, along the direction of the diffraction vector. Writing $\boldsymbol{\varepsilon}(\mathbf{x})$ for the local elastic strain tensor at the position $\mathbf{x}$ within the specimen, $\vec{k}$ for the unit vector parallel to the diffraction vector, $\langle\cdot\rangle_{V}$ for the volume average over $V$, and '. ' for the contracted (or scalar) product, $\varepsilon$ is then expressed by

$\varepsilon=\vec{k} \cdot\langle\boldsymbol{\varepsilon}(\mathbf{x})\rangle_{\Omega} \cdot \vec{k}$.

The macroscopic stress $\overline{\boldsymbol{\sigma}}$, i.e. the stress acting at the scale of the gauge volume, is given by an average over $V$ (and not $\Omega$ as above):

$\overline{\boldsymbol{\sigma}}=\langle\boldsymbol{\sigma}(\mathbf{x})\rangle_{V}$

with $\boldsymbol{\varepsilon}(\mathbf{x})=\mathbf{S}(\mathbf{x}): \boldsymbol{\sigma}(\mathbf{x})$, and $\mathbf{S}(\mathbf{x})$ the local elastic compliance at $\mathbf{x}$. Therefore, determining the macroscopic stress $\overline{\boldsymbol{\sigma}}$ for each gauge volume from our neutron measurements is not directly possible, as it requires estimating the tensor $\overline{\boldsymbol{\sigma}}$ from only one scalar value $\varepsilon$ measured on $\Omega$. Additional assumptions are needed.

On the other hand, the sources of elastic strain are multiple, as discussed above, and not all of them are associated with the presence of a macroscopic stress state. One of the sources is the tetragonal-monoclinic SPT. It induces a significant increase of the lattice volume. The second one is associated with the strongly anisotropic coefficients of thermal expansion of $\mathrm{ZrO}_{2}$ at the grain scale [26], creating internal stresses as the block is cooled down (the thermal expansion coefficients along lattice directions $\vec{b}$ or $\vec{c}$ differ by $\sim$ one order of magnitude). Write $\sigma_{\text {res }}(\mathbf{x})$ for the complex field of residual stress that develops due to these two phenomena in a polycrystalline $\mathrm{ZrO}_{2}$ specimen when it is very slowly cooled from a high temperature, i.e. with no temperature gradient in the specimen.

Lastly, when an initially stress-free polycrystal made of randomly oriented monoclinic grains is subjected to a mechanical test for a prescribed stress $\bar{\sigma}$, a heterogeneous stress field develops within the different grains, due to the anisotropy of the elastic behaviour at the grain scale. Indeed, the elastic stiffness $\mathbf{S}(\mathbf{x})$ at the grain level is strongly anisotropic [27], with a Young modulus $E$ ranging from $106 \mathrm{GPa}$ to $328 \mathrm{GPa}$ depending on the direction of loading with respect to the monoclinic crystal lattice. The local stress can be linked with the macroscopic one using a stress concentration tensor $\mathbf{B}(\mathbf{x})$

$\boldsymbol{\sigma}(\mathbf{x})=\mathbf{B}(\mathbf{x}): \overline{\boldsymbol{\sigma}}$ 
that only depends on the specimen's microstructure. Putting the above equations all together and adding the field of residual stress $\sigma_{\text {res }}$ due to the SPT and anisotropic dilation coefficients leads to

$\varepsilon=\vec{k} \otimes \vec{k}:\left[\langle\mathbf{S}: \mathbf{B}\rangle_{\Omega}: \overline{\boldsymbol{\sigma}}+\left\langle\mathbf{S}: \boldsymbol{\sigma}_{\text {res }}\right\rangle_{\Omega}\right]$

with $\otimes$ the dyadic product. A good estimate of the field of $\mathbf{B}(\mathbf{x})$ can be obtained with the self-consistent scale-transition model [28,29], which allows estimating the mechanical interactions between grains in a statistical way. This model assumes a microstructure made of randomly mixed $\mathrm{ZrO}_{2}$ grains and does not take into account the glassy phase, which is less stiff than $\mathrm{ZrO}_{2}$ (the Young modulus of borosilicate glass is $\sim 64 \mathrm{GPa}$ ).

In our experiment, when comparing the $2 \theta$ position of the Bragg peaks for the block with the $2 \theta$ position for the reference small cube, the second term in Eq. (6), associated with the residual stress $\sigma_{\text {res }}$, cancels out. This is valid of course only if the internal stress field $\sigma_{\text {res }}$ in the small reference cube and in the large block are similar, an assumption that should be valid as both have undergone the same thermal history. The self-consistent scheme allows estimating the so-called 'radiocrystallographic elastic constants' $\vec{k} \otimes \vec{k}:\langle\mathbf{S}: \mathbf{B}\rangle_{\Omega}$, which provide the lattice strain $\varepsilon$ for a given applied stress $\overline{\boldsymbol{\sigma}}$.

As already discussed, the experimental data do not allow estimating the direction of $\overline{\boldsymbol{\sigma}}$. We make the assumption that the macroscopic stress state is equibiaxial and parallel to the specimen surface (i.e. vanishing stress vector on the block surfaces, as for a free surface) although the measurements have been performed deeper inside the specimen where more complex stresses probably develop. To retrieve the elastic strain of $\sim-0.1 \%$ measured with neutrons for the ( $\overline{3} 11)$ plane along the direction of the diffraction vector, one would need a tensile stress on the order of $500 \mathrm{MPa}$. This value, however, should be considered as an upper bound, due to the presence of a continuous network of a compliant glassy phase around the $\mathrm{ZrO}_{2}$ crystals, an effect not taken into account in this calculation. This second phase, which carries less load than the stiff $\mathrm{ZrO}_{2}$, might lower the effective stress level in a given gauge volume. Moreover, in these materials a high density of small microcracks is observed by SEM within (sub)micrometric variants of monoclinic $\mathrm{ZrO}_{2}$. This might lower significantly the elastic stiffness and lead to smaller calculated values of the effective stress.

\section{Conclusion}

$\mathrm{ZrO}_{2}$ based ceramic refractory materials are subject to large internal stresses owing to their production method, their thermomechanical behaviour, and the structural phase transformations they undergo during cooling. These stresses can occur at different scales, making a multi-scale analysis with different residual strain measurement methods necessary to fully understand the phenomena. We have carried out diffraction experiments on the sub-micron level with Laue microdiffraction, conventional powder diffraction (0.5-2 mm of diffraction volume), and neutron diffraction. In this paper, we present the results of the last analysis.

The neutron diffraction experiments were realized on the SALSA beamline at ILL, Grenoble. With chosen collimator slit openings, an averaging for strain was done over relatively large gauge volumes $\left(4 \times 4 \times 20 \times \sin 2 \theta \mathrm{mm}^{3}\right.$, where $\left.2 \theta \approx 61^{\circ}\right)$. This allowed us to measure the level of strain at a macroscopic (millimetre) scale, i.e. a scale much larger than the characteristic size of the microstructure. Two different compositions of fused-cast blocks were measured along their ( 311$)$ planes. Lattice strains ranging between $-0.1 \%$ (compressive) and $+0.025 \%$ (tensile) have been obtained for $(\overline{3} 11)$ planes parallel to the block surface, for the block of composition ZB. The data do not allow estimating the stress tensor or the stress principal directions. Using instead a micromechanical approach and assuming an equibiaxial stress state parallel to the block surface yields stress values between $\sim+500 \mathrm{MPa}$ and $\sim-125 \mathrm{MPa}$, respectively. The block surface is globally under tension. These values could be an overestimate and might actually be lower due to the more compliant glassy matrix around $\mathrm{ZrO}_{2}$ and the existence of microcracks. The block of composition ZS shows a slightly smaller range of strain. There is a clear gradient from the surface to the centre across the large and small faces of both blocks. These gradients are usually symmetrical with respect to the centre of that particular face, showing rather isotropic conditions on planes normal to the casting axis.

The effect of the cooling conditions and the martensitic phase transformations on the development of the internal stresses were found to be multifaceted. Thermal gradients forcing volumetric expansions due to the phase transformations occurring at different times in different locations within the block should be regarded as a peculiar case of these materials. This is one of the most prominent causes of internal deformations at this scale within these blocks produced by casting. Our results at this point should be especially helpful for the mechanical modelling of this phenomenon.

\section{Acknowledgements}

This research was funded by the French National Research Agency under project ASZTech (ANR-12-RMNP-0007). The authors are thankful to Saint-Gobain CREE for supplying the materials and ILL staff (Thilo Pirling and Sergio Martinez Garcia) for their assistance during the measurements.

\section{Appendix A. Propagation of errors in the strain calculation with respect to a reference}

According to the strain calculation formula given in Eq. (2), there are two interdependent variables and their measurement introduces standard errors, $\Delta(2 \theta)$ and $\bar{\theta}_{0}$.

In order to obtain two independent variables, we can rewrite this equation by defining $\Delta(2 \theta)=\overline{2} \theta-\overline{2} \theta_{0}$ :

$\varepsilon=-\frac{1}{2} \cot \bar{\theta}_{0}(\Delta 2 \theta)=-\cot \bar{\theta}_{0}\left(\bar{\theta}-\bar{\theta}_{0}\right)$

where $\bar{\theta}$ is the position of the given reflection at a given point.

The general formula of error propagation for a function $\varepsilon\left(\bar{\theta}_{0}, \bar{\theta}\right)$ with independent variables is given by

$u_{\varepsilon}=\sqrt{\left(\frac{\partial \varepsilon}{\partial \bar{\theta}_{0}}\right)^{2} u_{\bar{\theta}_{0}}^{2}+\left(\frac{\partial \varepsilon}{\partial \bar{\theta}}\right)^{2} u_{\bar{\theta}}^{2}}$

where $u_{x}$ represents the standard error of a given variable $x$. Substituting Eq. (A.1) and differentiating yields

$u_{\varepsilon}=\sqrt{\left[\csc ^{2} \bar{\theta}_{0}\left(\bar{\theta}-\bar{\theta}_{0}\right)+\cot \bar{\theta}_{0}\right]^{2} u_{\bar{\theta}_{0}}^{2}+\left(-\cot \bar{\theta}_{0}\right)^{2} u_{\bar{\theta}}^{2}}$

In this paper, $u_{\bar{\theta}_{0}}$ has been obtained by different measurements of the reference samples and the values of $u_{\bar{\theta}}$ are the errors obtained by a Gaussian fit.

\section{References}

[1] P. Bardhan, R.N. McNally, Fusion-casting and crystallization of high temperature materials, J. Mater. Sci. 15 (10) (1980) 2409-2427, http://dx.doi. org/10.1007/BF00550743.

[2] C. Patapy, A. Proust, D. Marlot, M. Huger, T. Chotard, Characterization by acoustic emission pattern recognition of microstructure evolution in a fused-cast refractory during high temperature cycling, J. Eur. Ceram. Soc. 30 (15) (2010) 3093-3101, http://dx.doi.org/10.1016/j.jeurceramsoc.2010.07. 021.

[3] S.L. Cockcroft, J.K. Brimacombe, D.G. Walrod, T.A. Myles, Thermal stress analysis of fused-cast AZS refractories during production: Part I. Industrial 
study, J. Am. Ceram. Soc. 77 (6) (1994) 1505-1511, http://dx.doi.org/10.1111/ j.1151-2916.1994.tb09749.x.

[4] S.L. Cockcroft, J.K. Brimacombe, D.G. Walrod, T.A. Myles, Thermal stress analysis of fused-cast AZS refractories during production: Part II. Development of thermo-elastic stress model, J. Am. Ceram. Soc. 77 (6) (1994) 1512-1521, http://dx.doi.org/10.1111/j.1151-2916.1994.tb09750.x.

[5] E.H. Kisi, C.J. Howard, Crystal structures of zirconia phases and their inter-relation, in: E.H. Kisi (Ed.), in: Zirconia Engineering Ceramics: Old Challenges - New Ideas, vols. 153-154, Trans Tech Publications, Zurich, Switzerland, 1998, pp. 1-36, http://dx.doi.org/10.4028/www.scientific.net/ KEM.153-154.1.

[6] L. Petroni, M. Boussuge, D. Ryckelynck, Numerical simulation of the cooling-down of high-zirconia fused-cast refractories, J. Eur. Ceram. Soc. 32 (15) (2012) 3941-3947, http://dx.doi.org/10.1016/j.jeurceramsoc.2012.06. 004.

[7] The I12 beamline at Diamond and the 28-ID-2 beamline at NLSB-II synchrotron sources are specifically designed for XRD experiments using hard $\mathrm{X}$-rays (up to $150 \mathrm{keV}$ ). The penetration length into dense zirconia of a $100 \mathrm{keV} X$-ray beam is equal to $2.2 \mathrm{~mm}$.

[8] P.J. Withers, P.J. Webster, Neutron and synchrotron X-ray strain scanning, Strain 37 (1) (2001) 19-33, http://dx.doi.org/10.1111/j.1475-1305.2001. tb01216.x.

[9] F. Tang, C.R. Hubbard, G. Sarma, J. Keiser, Residual strain distribution in bent composite boiler tubes, Mater. Sci. Eng. A 437 (1) (2006) 83-87, http://dx.doi. org/10.1016/j.msea.2006.04.050.

[10] H. Hao, D. Maijer, R. Rogge, Investigation of residual strains by neutron diffraction in an AZ31 direct chill cast billet, NDT\&E Int. 42 (8) (2009) 704-712, http://dx.doi.org/10.1016/j.ndteint.2009.06.006.

[11] L. Edwards, M. Fitzpatrick, P. Irving, An integrated approach to the determination and consequences of residual stress on the fatigue performance of welded aircraft structures, J. ASTM Int. 3 (2) (2006) 1-17.

[12] P. Bouchard, D. George, J. Santisteban, G. Bruno, M. Dutta, L. Edwards, E. Kingston, D. Smith, Measurement of the residual stresses in a stainless steel pipe girth weld containing long and short repairs, Int. J. Press. Vessels Pip. 82 (4) (2005) 299-310, http://dx.doi.org/10.1016/j.ijpvp.2004.08.008.

[13] C. Patapy, F. Gouraud, M. Huger, R. Guinebretière, B. Ouladiaff, D. Chateigner, T. Chotard, Investigation by neutron diffraction of texture induced by the cooling process of zirconia refractories, J. Eur. Ceram. Soc. 34 (15) (2014) 4043-4052, http://dx.doi.org/10.1016/j.jeurceramsoc.2014.05.027.

[14] C. Patapy, M. Huger, R. Guinebretière, N. Gey, M. Humbert, A. Hazotte, T. Chotard, Solidification structure in pure zirconia liquid molten phase, J. Eur. Ceram. Soc. 33 (2) (2013) 259-268, http://dx.doi.org/10.1016/j.jeurceramsoc. 2012.08.021.
[15] M. Humbert, N. Gey, C. Patapy, E. Joussein, M. Huger, R. Guinebretiere, T. Chotard, A. Hazotte, Identification and orientation determination of parent cubic domains from electron backscattered diffraction maps of monoclinic pure zirconia, Scr. Mater. 63 (2010) 411-414, http://dx.doi.org/10.1016/j. scriptamat.2010.04.039.

[16] V.F. Sears, Neutron scattering lengths and cross sections, Neutron News 3 (3) (1992) 29-37.

[17] W. Kraus, G. Nolze, POWDER CELL - a program for the representation and manipulation of crystal structures and calculation of the resulting X-ray powder patterns, J. Appl. Crystallogr. 29 (3) (1996) 301-303, http://dx.doi org/10.1107/S0021889895014920.

[18] D. Richard, M. Ferrand, G.J. Kearley, LAMP, the Large Array Manipulation Program.

[19] V. Klosek, Internal report of the Laboratoire Leon Brillouin. Tech. rep., Laboratoire Leon Brillouin, Saclay, France, 2014.

[20] T. Williams, C. Kelley, gnuplot. https://sourceforge.net/projects/gnuplot/.

[21] U. Chandra, A. Ahmed, Stress analysis, in: K. Yu (Ed.), Modeling for Casting and Solidification Processing, Marcel Dekker Inc., New York, 2002, pp. 55-95.

[22] I. Noyan, J. Cohen, Residual Stress: Measurement by Diffraction and Interpretation, Springer-Verlag, 1987.

[23] N. Letouzé, R. Brenner, O. Castelnau, J.-L. Béchade, M.-H. Mathon, Residual strain distribution in Zircaloy-4 measured by neutron diffraction and estimated by homogenization techniques, Scr. Mater. 47 (2002) 595-599.

[24] T. Bretheau, O. Castelnau, Rayons X et Matière (RX2006), Hermès, 2006. Ch. Les contraintes résiduelles: d'où viennent-elles? Comment les caractériser? 2006, pp. 123-154.

[25] D. Faurie, O. Castelnau, R. Brenner, P. Renault, E.L. Bourhis, P. Goudeau, In situ diffraction strain analysis of elastically deformed polycrystalline thin films, and micromechanical interpretation J. Appl. Cryst. 42 (2009) 1073-1084.

[26] A. Sibil, T. Douillard, C. Cayron, N. Godin, M. R'mili, G. Fantozzi, Microcracking of high zirconia refractories after t-m phase transition during cooling: an EBSD study, J. Eur. Ceram. Soc. 31 (9) (2011) 1525-1531, http://dx.doi.org/10 1016/j.jeurceramsoc.2011.02.033.

[27] S.-K. Chan, Y. Fang, M. Grimsditch, Z. Li, M.V. Nevitt, W.M. Robertson, E.S. Zouboulis, Temperature dependence of the elastic moduli of monoclinic zirconia, J. Am. Ceram. Soc. 74 (1991) 1742-1744.

[28] A.V. Hershey, The elasticity of an isotropic aggregate of anisotropic cubic crystals, J. Appl. Mech. 21 (1954) 236-240.

[29] E. Kröner, Self-consistent scheme and graded disorder in polycrystal elasticity, J. Phys. F: Met. Phys. 8 (1978) 2261-2267. 